\title{
A One Year Study of Pancreatic Fluid Collections at a Tertiary Care Hospital of South India
}

\author{
Suresh Kumar ${ }^{1}$, Kishore Kumar Markapuram ${ }^{2}$, Srinivas M G ${ }^{3}$, Ramakrishna Baru ${ }^{4}$, Sreeram Satish ${ }^{5}$ \\ ${ }^{1}$ Assistant professor, Department of Radiology, ${ }^{2}$ Associate professor, Department of General Surgery, ${ }^{3}$ Associate professor, \\ Department of Medical Gastroenterology, ${ }^{4}$ Professor and HOD department of Radiology, ${ }^{5}$ Professor and HOD, Department \\ of General Surgery, Narayana Medical College, Chinthareddypalem, Nellore, Andhra Pradesh, India
}

Corresponding author: Dr Kishore Kumar Markapuram, Associate professor, Department of General Surgery, Narayana Medical College, Chinthareddypalem, Nellore, Andhra Pradesh, India

DOI: $10.21276 /$ ijcmsr.2018.3.2.23

How to cite this article: Suresh Kumar, Kishore Kumar Markapuram, Srinivas M G, Ramakrishna Baru, Sreeram Satish. A one year study of pancreatic fluid collections at a tertiary care hospital of South India. International Journal of Contemporary Medicine Surgery and Radiology. 2018;3(2):B92-B96.

\section{A B S T R A C T}

Introduction: Pancreatic fluid collections are categorised into pseudocysts (PC) and walled-off necrosis. Drainage is required in cases when there are mass effects like obstruction or infection. Endoscopic ultra sounded drainage is now firmly established as the best option for drainage of walled off pseudocysts. The study outlines the procedure with respect to its technical success, recurrence rate and complications.

Material and methods: A cross sectional study was conducted for 6 months after approval of ethical committee. Endoscopic ultrasound drainage was performed on 50 selected cases and all the features were noted with follow up. The data was statistically analyzed by using SPSS software and P value $<0.01$ was considered significant.

Results: 50 cases were placed in two Groups of A with pancreatic pseudocyst and Group B with Pancreatic abscess. Mean age of study group was $53 \pm 14$ years and mean hospital stay of the total cases in the study was $10 \pm 2.8$ days with technical success rate of $88 \%$. No relapses were observed in the study. $4 \%$ was the mortality in the study with all in Group B. The treatment procedures was EUS-D alone in 31 (62\%) of cases and EUS-D with stenting in 16\% followed by percutaneous drainage in $14 \%$ and necrosectomy in $8 \%$ of cases.

Discussion: EUS-d is the preferred modality for drainage of pancreatic fluid collections in asymptomatic individuals. The plan of strategy in patients with walled off pancreatic necrosis can be drained by either plastic or metallic stents is an ongoing work with multiple results favouring the plastic stents in drainage. We suggest that patients with thick walled collections should be managed surgically until development of new devices for wall penetration is developed.

Key words: Pancreatic Pseudocyst, Endoscopic Ultrasound Drainage, Pancreatic Abscesses, Necrosectomy.

\section{INTRODUCTION}

Pancreatic fluid collections are categorised into pseudocysts (PC) and walled-off necrosis (WON). A pancreatic pseudocyst is a circumscribed collection of fluid rich in pancreatic enzymes, blood and necrotic tissue, typically located in the lesser sac of the abdomen. As per the Atlanta classification, the cause of acute pseudocysts may be acute trauma or acute pancreatitis while chronic pseudocysts arise due to chronic pancreatitis, and usually lack an antecedent episode of pancreatitis. ${ }^{1}$ Drainage is required in cases when there are mass effects like obstruction or infection. Infection of a pseudocyst leads to formation of a pancreatic abscess. Abscesses associated with infection can develop systemic symptoms like high fever and sepsis associated with poor outcome. Most of the cases are asymptomatic and require no treatment except watchful nature but treatment is essential in cases where the pancreatic cysts are associated with abscess formation and infection, size exceeding $5-8 \mathrm{~cm}$ in diameter after 4-8 weeks of onset of acute pancreatitis. Various techniques of drainage have been performed in the past with surgical drainage, trans- papillary drainage and transmural conventional endoscopic drainage. ${ }^{2}$ Treatment options for these pseudocysts are surgical drainage which can be performed by standard cut (open surgical drainage) or by key hole surgery (Laparoscopic surgical drainage). Endoscopic ultra sounded drainage is now firmly established as the best option for drainage of walled off pseudocysts. Decompression of the pseudocyst by internal or percutaneous drainage is practised in symptomatic patients and internal drainage can be done by endoscopic or surgical cystogastrostomy. This procedure creates a fistula between the pseudocyst and the stomach with placement of a stent with technical success and treatment success rate of $89-100 \%$ and $82-100 \% .^{3}$ Current clinical management studies in pancreatic pseudocysts favour endoscopic ultrasound drainage which has better results compared to percutaneous drainage, non guided endoscopic drainage and surgical drainage. ${ }^{4}$

The aim of the present study was to establish the safety of 
endoscopic ultrasound drainage of pancreatic fluid collections and to also identify the selection criteria of the patients who are benefited by this method. The study soutlines the procedure with respect to its technical success, recurrence rate and complications.

\section{MATERIAL AND METHODS}

A cross sectional study was conducted at a tertiary care hospital for a period of one year from November 2015 to October 2016. The study was conducted by department of General surgery in association with department of Radiology on patients identified with pancreatic fluid collections and admitted in the ward of general surgery. The study was approved by the institutional ethical committee and all the patients who participated in the study were informed about the study protocol and written consent was obtained from all the cases. The demographic data of the cases in the study was noted in a separate pre designed questionnaire sheet and also included clinical history, laboratory data, and radiological findings. All the cases in the study were performed ultra sonogram of complete abdomen, computed tomography (CT) and in necessary cases a magnetic resonance imaging scan (MRI) and detailed ERCP in doubtful cases. A pseudocyst was defined as a fluid collection in pancreatic or peripancreatic area that had a well defined wall and contained no solid debris or recognizable parenchymal necrosis.

\section{Inclusion criteria}

Pseudocyst measuring $\geq 6 \mathrm{~cm}$ and located adjacent to stomach History of acute or chronic pancreatitis

Diagnosis of pancreatic pseudocyst by CT criteria

Pain due to pancreatitis requiring analgesics

Gastric outlet or bile duct obstruction due to pseudocyst

\section{Exclusion criteria}

Age $<18$ years or $>80$ years.

Contraindications to surgery and endoscopic drainage

Pregnancy

Identified necrosis of pancreasis on CT

Pregnancy

Multiple or Multiloculated pseudocyst

Endoscopic ultrasound (EUS) drainage was performed by a senior experienced surgeon and all the patients were screened by transabdominal ultrasound. Communication of pseudocysts with pancreatic duct was ruled out by performing MRI and ERCP in doubtful cases. EUS was performed to evaluate the following criteria: a well defined wall, identification of vessels in needle tract, distance between pseudocyst and gut wall, no vascular aneurysms, and no other cystic lesions in pancreas. The following features were noted in cases of pseudocysts: thickness and vascularization of the cystic wall, best window for access of drainage, portal hypertension, and presence of septa and aspect of fluid in the cyst.

\section{Drainage protocol}

All the procedures were conducted by using a therapeutic linear array echo endoscope with patient in left lateral position and administering IV ciprofloxacin. Once the pseudocyst was identified it was accessed using a 19 gauge needle and gastric wall was dilated up to $15 \mathrm{~mm}$ using a wired balloon. Two plastic stents were deployed for drainage of pseudocyst contents into the stomach. If any pancreatic duct leakage was observed a $5 \mathrm{f}$ pancreatic duct stent was placed to bridge the site of leak or stricture.

Pancreatic abscess: In cases identified by abscess a EUS guided direct puncture was performed to create a fistula similar to management of pseudocyst. The fistula was dilated with a $20 \mathrm{~mm}$ controlled radial expansion balloon. In cases of inadequate treatment puncture was done in a different route or a percutaneous fistula was created under ultrasound guidance or CT. IV line was set up and antibiotic infusions were given.

Technical success of the cases was defined as ability to place a transmural stent to drain a pancreatic pseudocyst. Technical failure of the drainage was considered when drainage was not achieved. Treatment success was defined after complete resolution or decrease in size of the cyst after 3 months follow up with resolution of clinical symptoms. Outcomes were recorded as treatment success, treatment success in long term follow up and development of immediate and late complications.

\section{STATISTICAL ANALYSIS}

The data was entered in Microsoft excel sheet and tabulated. The corrected data was analyzed using SPSS software Version for Windows 10 . The age, sex ratio was calculated. $\mathrm{P}$ value $<0.001$ was considered significant.

\section{RESULTS}

The study was carried out for a period of one year from November 2106 to October 2017 and a total of 110 patients were screened and 50 patients with pancreatic fluid collection were included. Cases suspicious of other causes like tumours were excluded from the study. All the cases included in the study were followed till 24 months (range 6-24 months). Patient's characteristics are shown in Table-1. The total 50 cases in the study were categorized into Group A containing Pancreatic abscess (14/50, 28\%) and Group B with pancreatic pseudocyst (36/50, 72\%). Out of 50 cases, males were predominant with 32 number and 18 females. The mean age of the total study group was $53 \pm 14$ years, Group A $51.14 \pm 10$ years and Group B was $56 \pm 9$ years. $78 \%$ of cases were having acute pancreatitis as etiological factor and $22 \%$ as chronic pancreatitis. Tail and body were the common locations of pancreatic fluid collections in the study with 22 cases each in tail and body and 6 cases in the head of the pancreas. Endoscopic bulging was observed in 16\% of cases in the study with equal distribution in both groups. The mean size of the collection in the study was $71.14 \pm 14 \mathrm{~mm}$ with maximum size in Group B $(72.08 \pm 20 \mathrm{~mm})$ and in group A with $65.14 \pm 14.7 \mathrm{~mm}$. In 33 cases $(66 \%)$ the size of the organ was $>6 \mathrm{~cm}$ in diameter. In $30 \%$ of cases the thickness of the fluid wall was $\geq 2 \mathrm{~mm}$ and $14 \%$ of cases had septa in the collection. A clear fluid was observed in 32 cases whereas 18 cases had thick purulent pus in the collection. In $24 \%$ of cases signs of portal hypertension were observed with majority in Group B cases (22.22\%). [Table-1]

With reference to the aetiology of development of pancreatic 


\begin{tabular}{|l|c|c|c|}
\hline & Group A (Abscesses) & Group B (Pseudocyst) & Total \\
\hline No of Patients & $14(28 \%)$ & $36(72 \%)$ & $53 \pm 14$ \\
\hline Age (Years) & $\begin{array}{c}51.14 \pm 10 \\
(41-64)\end{array}$ & $\begin{array}{c}56 \pm 9 \\
(44-70)\end{array}$ & $32: 18$ \\
\hline Male : Female Ratio & $8: 6$ & $24: 12$ & 38 \\
\hline Aetiology & & & 12 \\
\hline Acute & 10 & 8 & $6: 22: 22$ \\
\hline Chronic & 4 & $4: 16: 16$ & $8(16)$ \\
\hline Location (Head: Body: tail) & $4: 6: 6$ & $4(8)$ & $71.14 \pm 14$ \\
\hline Endoscopic bulging & $65.14 \pm 14.7$ & $72.08 \pm 20$ & 33 \\
\hline Size (mm) & $8(16)$ & $25(12.5 \%)$ & $34: 16$ \\
\hline Size $>6 c m$ in Diameter & $10: 04$ & $24: 12$ & $15(30 \%)$ \\
\hline Anechoic: Hypoechoic & $4(28.57 \%)$ & $11(30.56 \%)$ & $7(14 \%)$ \\
\hline Thick wall (>2mm) & $2(14.28 \%)$ & $5(13.89 \%)$ & $32: 18$ \\
\hline Septa & $0: 14$ & $32: 4$ & $12(24 \%)$ \\
\hline Fuid aspect (clear:Pus) & $4(28.57 \%)$ & $8(22.22 \%)$ & \\
\hline Portal hypertension signs & Table-1: Patients characteristics in study & \\
\hline
\end{tabular}

\begin{tabular}{|l|c|c|c|c|}
\hline Parameter & Total ( $\mathbf{n = 5 0 )}$ & $\begin{array}{c}\text { Group A } \\
\text { (Abscesses) ( } \mathbf{n = 1 4 )}\end{array}$ & $\begin{array}{c}\text { Group B } \\
\text { (Pseudocysts) (N=36) }\end{array}$ & 'P' value \\
\hline Place of Drainage & & & & \\
\hline Stomach & $38(76 \%)$ & $10(71.42 \%)$ & $28(77.78 \%)$ & 1.021 \\
\hline Duodenum & $12(24 \%)$ & $4(28.57 \%)$ & $8(22.22 \%)$ & 0.98 \\
\hline Median Hospital stay (Days) & $10 \pm 2.8$ & $11 \pm 1.6$ & $10 \pm 0.8$ & Not significant \\
\hline Technical success (n, \%) & $44(88 \%)$ & $12(85.71 \%)$ & $32(88.89 \%)$ & Not significant \\
\hline Clinical Resolution after successful drainage $(n, \%)$ & $47(94 \%)$ & $13(92.85 \%)$ & $34(94.45 \%)$ & 1.121 \\
\hline Need for surgery after successful drainage $(n, \%)$ & $3(6 \%)$ & $1(7.14 \%)$ & $2(5.56 \%)$ & Not significant \\
\hline Rate of Recurrence & 0 & 0 & 0 & - \\
\hline Immediate complications & $4(8 \%)$ & $1(7.14 \%)$ & $3(8.33 \%)$ & Not significant \\
\hline Late complications & $4(8 \%)$ & 0 & $4(11.11 \%)$ & Not significant \\
\hline Deaths & $2(4 \%)$ & 0 & $2(5.56 \%)$ & \\
\hline \multicolumn{2}{|c|}{ Table-2: Results of EUS guided drainage } & \\
\hline
\end{tabular}

\begin{tabular}{|l|c|c|c|c|c|}
\hline Treatment & N (\%) & SEX- M:F & Acute & Chronic & Mean size(CM) \\
\hline EUS-Drainage & $31(62)$ & $21: 10$ & 28 & 3 & 10.1 \\
\hline +EPS & $8(16)$ & $5: 3$ & 5 & 3 & 12.4 \\
\hline +Percutaneous drainage & $7(14)$ & $3: 4$ & 4 & 3 & 13.5 \\
\hline +Necrosectomy & $4(8)$ & $3: 1$ & 38 & 12 & 16.2 \\
\hline Total & 50 & $32: 18$ & 3 & 12.24 \\
\hline \multicolumn{2}{|l}{ Table-3: Basic characteristics of endoscopic treatment for Pseudocyst and abscesses, * EPS- endoscopic pancreatic stent } \\
\hline
\end{tabular}

fluid collections, alcohol abuse was the commonest (78\%) followed in order by gallstones (10\%), post-trauma (6\%) and idiopathic in $6 \%$ of cases.

Table -2 summarizes the findings of EUS drainage in the study. The drainage was performed via the stomach in $76 \%$ of cases and in duodenum in $24 \%$ of cases. The mean hospital stay of the total cases in the study was $10 \pm 2.8$ days with maximum in Group A cases ( $11 \pm 1.6$ days).

Technical success: The overall success rate in the study was $88 \%$ (44/50) with $85.71 \%$ in group A and $88.89 \%$ in Group B. 2 cases in group A registered failure due to development of immediate complication and 4 cases in group B due to a thick cystic wall in cases of chronic pancreatitis. No statistical significance was observed in both the groups regarding technical success. ( $\mathrm{P}$ value $<0.001)$ In 47 cases (94\%) a complete resolution of the collection was observed after drainage and only 3 cases (6\%) required surgical management. $94.45 \%$ of cases in group B had successful drainage and $92.85 \%$ in Group A. However the cause of non resolution needs to be evaluated in cases which required surgical management.

In our study none of the cases developed relapses. Three cases (8.33\%) and one case in group A (7.14\%) developed immediate complications. One case of pneumoperitoneum was successfully treated by surgical management and bleeding from two cases of portal hypertension which was managed conservatively. None of the cases in Group A developed late complications whereas four cases (11.11\%) from group B developed late complications with two cases 
of parietal bleeding in stent placement sites and in other two abscess formation at stent placement site.

The mortality in the total study was $4 \%$ and all were from Group B. The cause of death in two cases was parietal bleeding associated with portal hypertension and also was related with pulmonary COPD. In the total study no statistical significance was associated with any factors in between Group A and Group B. (P value>0.01)

The treatment procedures was EUS-D alone in 31 (62\%) of cases and EUS-D with stenting in 16\% followed by percutaneous drainage in $14 \%$ and necrosectomy in $8 \%$ of cases. [Table-3]

\section{DISCUSSION}

Pancreatic fluid collections develop after surgical trauma, acute or chronic pancreatitis secondary to leakage or liquefaction of pancreatic tissue. These collections can form as a cyst or these collections in the cyst may get infected or infected necrotic tissue resulting in formation of an abscess which acts as a predisposing factor for development of sepsis or leading to multi organ failure. In most of the studies mentioned previously these pseudocysts disappear in course of time within 6-8 weeks in $40 \%$ of cases with a complication rate of $20 \% .^{5} \mathrm{With}$ increase in time the rate of disappearance was reduced with increase in chance of development of complications. At time of 13 weeks the rate of disappearance was $0 \%$ and the chance of development of complications was $75 \%$ as mentioned in the study of Bradley et al in his findings. ${ }^{6}$ However findings in the study of Yeo CJ et al reported contrast findings with less chance of complications with progression of time in cases of pancreatic pseudocysts. ${ }^{7}$ Most of the studies reported that mortality rate in cases of necrotizing pancreatitis as $50 \%$ in untreated cases resulting from secondary factors like sepsis and multi-organ failure. Trans-papillary duct stenting usually was an accepted first choice of management in older days but withdrawn due to higher chances of recurrence rate. The initial resolution success rate of this procedure was not $100 \%$.

Roger's et al described Endoscopic ultrasound as a procedure for internal drainage in 1975 which was later modified by Grimm et al in 1992 as Endoscopic ultrasound drainage(EUS-D) which was widely accepted as a first line choice in cases of pancreatic pseudocysts., ${ }^{8,9}$ Other treatment modalities were percutaneous drainage, stenting, surgical cystogastrostomy and necrosectomy. Endoscopic conventional transmural drainage was limited to cases of pseudocysts with no bulging onto gut wall, in cases of portal hypertension, and in cases where difficulty is encountered in positioning of endoscope.

In our study we analyzed the safety of performing EUS-D and other management modalities in cases of pancreatic pseudocysts and abscesses. Our success rate in the study was $88 \%$ with 0 recurrence rate which was quite similar to studies of Bapaye A et al but with recurrence rate of $1 \%$ in his study and contrast to findings of Dhir $\mathrm{V}$ et al who reported a success rate of $98 \%$ in his study with a recurrence rate of $2 \% .{ }^{10,11}$ Thick wall of the collection was an impeding factor in the study and in patients with uncomplicated pseudocyst sliding of the cystosome on the wall surface and difficult wall penetration were other factors.At present there are only a few studies focussing on treatment studies with pancreatic pseudocysts, abscesses and necrotising pancreatitis treated by EUS-D and necrosectomy. In our study 4 patients underwent necrosectomy with 3 males and 3 chronic cases of pancreatitis and all the cases were having alcoholic abuse. So in our study the death was unrelated to necrosectomy but because of COPD in this case. Findings of our study were similar to findings of Seifert $\mathrm{H}$ et al who reported the same in cases of necrosectomy in his study. ${ }^{12}$

A retrospective study conducted by Varadarajulu et al compared EUS-D with surgical cystogastrostomy observed and reported that surgical intervention was associated with less hospital stay and low cost. But these finding were contrary to the findings in our study where hospital stay was less in cases of EUS-D. ${ }^{13}$ Percutaneous drainage is performed by puncturing the abdominal wall using CT or EUS guidance. In our study it was performed in 7 cases where the target lesion was located far from the gastro intestinal tract. This procedure is associated with development of fistula in the pancreas. In our study no such complication was observed which is similar to the findings of Mukai $\mathrm{S}$ et al. ${ }^{14} \mathrm{Few}$ of the other prospective studies conducted by Kahaleh et al and Park et al compared endoscopic drainage with EUS-D and found that there was no significant therapeutic difference in the procedures. ${ }^{15,16}$ Many of the studies reported that percutaneous drainage has been associated with higher rates of re interventions and longer lengths of stay in hospital compared with EUS-D and surgical cystogastrostomy. The findings of their studies correlate with findings of our study. In our study, we concluded that EUS-drainage appeared advantageous in drainage of pseudocysts located adjunct to the stomach or duodenum.

In our study, 8 cases underwent endoscopic pancreatic stenting (EPS) with 5 acute cases and 3 chronic cases. Fully covered self expandable metallic stents were used in these cases and had a better outcome. A few studies reported the success rate of $75-91 \%$ in cases of pancreatic fluid collections. In our study the success rate was $80 \%$ with 2 cases of failure because of bleeding and fistula as a secondary complication. Findings of our study were consistent with the findings of Ang et al who reported $100 \%$ success rate in his observational study without recurrences. ${ }^{17}$

\section{CONCLUSION}

To conclude, our study clearly highlights the success of EUS-D in comparison with surgical cystogastrostomy and necrosectomy in management of pancreatic fluid collections i.e, pancreatic pseudocysts and abscesses. EUS-d is the preferred modality for drainage of pancreatic fluid collections in asymptomatic individuals. The plan of strategy in patients with walled off pancreatic necrosis can be drained by either plastic or metallic stents is an ongoing work with multiple results favouring the plastic stents in drainage. Few other studies prefer necrosectomy in cases with severe complications like sepsis and multi-organ failure. Disadvantage in necrosectomy is multiple sessions are required as only small amount of material can only be removed in one session. Devices capable of removing more 
amount of necrotic material to be developed. Most of the studies suggest fluoroscopic guidance in EUS-D. However the rate of success without fluoroscopy was comparable with data published from other studies. We suggest that patients with thick walled collections should be managed surgically until development of new devices for wall penetration is developed.

\section{REFERENCES}

1. Andrén-Sandberg A, Dervenis C. Pancreatic pseudocysts in the 21st century. Part I: Classification, pathophysiology, anatomic considerations and treatment. JOP. 2004;5(1):8-24.

2. Binmoeller KF, Seifert H, Walter A, Soehendra N. Transpapillary and transmural drainage of pancreatic pseudocysts. Gastrointest Endosc 1995; 42(3): 219-224.

3. AzarRR,OhYS,Janee EM, et al.Wire-guided pancreatic pseudocyst drainage by using a modified needle knife and therapeutic echo endoscope. Gastrointest Endosc 2006;63(5):688-692.

4. Nealon WH, Walser E. Main pancreatic ductal anatomy can direct choice of modality for treating pancreatic pseudocysts (surgery versus percutaneous drainage). Ann Surg. 2002;235(2):751.

5. Bradley EL 3rd, Clements JL Jr, Gonzalez AC. The natural history of pancreatic pseudocysts: a unified concept of management. Am J Surg 1979; 137(4): 13541.

6. Bradley EL 3rd, Howard TJ, van Sonnenberg E, etal. Intervention in necrotizing pancreatitis: an evidencebased review of surgical and percutaneous alternatives. J Gastrointest Surg 2008; 12(6): 634-9.

7. Yeo CJ, Bastidas JA, Lynch-Nyhan A, et al. The natural history of pancreatic pseudocysts documented by computed tomography. Surg Gynecol Obstet 1990; 170(3): 411-7.

8. Grimm H, Binmoeller KF, Soehendra N.Endosonography-guided drainage of a pancreatic pseudocyst. Gastrointest Endosc 1992; 38(1): 170-1.

9. Rogers BH, Cicurel NJ, Seed RW. Transgastric needle aspiration of pancreatic pseudocyst through an endoscope. Gastrointest Endosc 1975; 21(5): 1334.

10. Bapaye A, Dubale NA, Sheth KA et al. Endoscopic ultrasonography-guided transmural drainage of walledoff pancreatic necrosis: Comparison between a specially designed fully covered bi-flanged metal stent and multiple plastic stents. Dig Endosc. 2017; 29(2): 10410.

11. Dhir V, Teoh AY, Bapat M, Bhandari S, Joshi $\mathrm{N}$, Maydeo A.EUS-guided pseudocyst drainage: prospective evaluation of early removal of fully covered self-expandable metal stents with pancreatic ductal stenting in selected patients. Gastrointest.Endosc. 2015; 82(6): 650-7.

12. Seifert H, Biermer M, Schmitt W, et al. Transluminal endoscopic necrosectomy after acute pancreatitis: a multicentre study with long-term follow-up (the GEPARD Study). Gut 2009; 58(4): 1260-6.

13. Varadarajulu S, Lopes TL, Wilcox CM, et al. EUS versus surgical cyst-gastrosomy for management of pancreatic pseudocysts. Gastrointest Endosc 2008;68(4): 649-55.
14. Mukai S, Itoi T, Sofuni A, et al. Expanding endoscopic interventions for pancreatic pseudocyst and walled-off necrosis. J Gastroenterol. 2015;50(5):211-20.

15. Park DH, Lee SS, Moon SH, et al. Endoscopic ultrasound-guided versus conventional transmural drainage for pancreatic pseudocysts: a prospective randomized trial. Endoscopy 2009; 41(4): 842-8.

16. Kahaleh M, Shami VM, Conaway MR, et al.Endoscopic ultrasound drainage of pancreatic pseudocyst: a prospective comparison with conventional endoscopic drainage. Endoscopy 2006;38(3): 355-9.

17. Ang TL, Kongkam P, Kwek ABE, Orkoonsawat P, Rerknimitr R, Fock KM. A two-center comparative study of plastic and lumen apposing large diameter selfexpandable metallic stents in endoscopic ultrasound guided drainage of pancreatic fluid collections. Endosc. Ultrasound 2016; 5(2): 320-7.

Source of Support: Nil; Conflict of Interest: None

Submitted: 25-04-2018; Accepted: 29-05-2018; Published online: 05-06-2018 